\title{
Gauge fields on truncated Heisenberg space
}

\author{
Maja Burić* ${ }^{\dagger}$ \\ Faculty of Physics, University of Belgrade \\ Studentski trg 12, SR-11001 Belgrade \\ E-mail: majab@ipb.ac.rs
}

Harald Grosse

Department of Physics, University of Vienna

Boltzmanngasse 5, A-190 Vienna

E-mail: harald.grossedunivie.ac.at

\section{John Madore}

Laboratoire de Physique Théorique, Université de Paris-Sud

Bâtiment 211, F-91405 Orsay

E-mail: madoredth.u-psud.fr

Corfu Summer Institute on Elementary Particles and Physics - Workshop on Non Commutative Field Theory and Gravity

September 8-12, 2010

Corfu, Greece

\footnotetext{
*Speaker.

${ }^{\dagger}$ Research done within Project No. 141036 of MNTR, Serbia.
} 


\section{Motivation}

Noncommutativity of coordinates was introduced to physics with the idea that it could improve renormalizability of quantum field theory, [1]. Intuitively speaking, this idea can be understood as follows: if coordinates do not commute, the corresponding uncertainty relations give a lower bound to coordinate measurements; this bound acts as a natural UV regulator to divergent integrals. Or similarly: division of space into cells analogous to the partition of phase space in quantum mechanics is not rigid, therefore in principle it provides with discretization which can be adjusted to symmetries; such discretization could regularize a theory without breaking its symmetries.

These ideas remain to a great extent our motivation today. However, in spite of many successes of noncommutative physics and mathematics such as for example a beautiful mathematical framework of noncommutative geometry or new noncommutative models in particle physics, the question of renormalizability is not solved satisfactorily yet.

This is not surprising. Replacement of the spacetime which is a smooth manifold with an algebra of operators changes the mathematical structures of the 'underlying manifold', and the 'space of functions' on it very much. Nonetheless, we wish to transfer most of the notions we are accustomed to to the noncommutative description of physics. For example, in order to speak of fields and field equations we need a notion of smoothness, so we wish to introduce the differential and the derivations on a 'noncommutative space'. We also need the integral, in order to obtain the classical lagrangian description of fields which includes the action and the variational principle. We wish to describe geometry of the background noncommutative space, flat or curved, and to relate it to gravity. We wish to keep physically very important notion of symmetry in an unchanged or deformed form: besides global symmetries we would like to implement gauge symmetries and diffeomorphisms. Finally, we wish to quantize classical noncommutative fields by one of the known methods (and obtain finite results). To all this we add (a natural) requirement of existence of the (correct) commutative limit, at classical and perhaps also at the quantum level. This is a very ambitious, perhaps an 'overcomplete' programme, and it is very likely that we will have to relax or modify some of the listed requirements. Not only because all these conditions might not be compatible or independent, but also because we in principle expect to obtain new effects so the system should not be overconstrained in advance.

In the context of renormalizability one of the most interesting models is the Grosse-Wulkenhaar model. It is given by the action $[2,3]$

$$
S=\int \frac{1}{2} \partial_{\mu} \varphi \partial^{\mu} \varphi+\frac{m^{2}}{2} \varphi^{2}+\frac{\Omega^{2}}{2} x^{\mu} \varphi x_{\mu} \varphi+\frac{\lambda}{4 !} \varphi^{4}
$$

where scalar field $\varphi(x)$ is defined on two- or four-dimensional noncommutative space with constant noncommutativity

$$
\left[x^{\mu}, x^{v}\right]=i \theta^{\mu v}
$$

and $\mathrm{x}_{\mu}$ is defined as $\mathrm{x}_{\mu}=\theta_{\mu \nu}^{-1} x^{\nu}$. We will here discuss two-dimensional space, $x^{1}=x, x^{2}=y$ and denote

$$
[x, y]=i \varepsilon \mu^{-2},
$$


which gives the usual Heisenberg algebra. Grosse-Wulkenhaar model (1.1) is fully renormalizable. Being one of a few renormalizable models, a straighforward question to ask is: which feature distiguishes is, that is, what exactly ensures its renormalizability?

One answer was proposed by Langmann and Szabo, [4], who suggested that the reason of renormalizability is a special symmetry which the model posesses, the Langmann-Szabo duality. It relates short and long distances, that is, exchanges coordinates $x^{\mu}$ with momenta $p^{\mu}=-i x^{\mu}$, or commutators $\left[x_{\mu},\right]$ with anticommutators $\left\{x_{\mu},\right\}$. One can think that in a way this property supresses the UV/IR mixing. We on the other hand noticed in [7] that the Grosse-Wulkenhaar model is closely related to a model defined on a curved noncommutative space generated by finite matrices. Namely, the first proof of renormalizability of the Grosse-Wulkenhaar model was done in the 'matrix base' in which coordinates and fields are represented by infinite matrices. Truncation to $n \times n$ matrices and the consequent limit $n \rightarrow \infty$ allowed various estimates and eventually the proof of renormalizability. Thus, one can argue that relation of the Grosse-Wulkenhaar model with a matrix one gives the 'necessary amount' of discretization: matrices and their corresponding noncommutative geometry act as a regulator. Here we develop this idea further by analyzing $U_{1}$ gauge fields defined on the same matrix space.

\section{Truncated Heisenberg algebra}

The truncated Heisenberg algebra is defined by relations

$$
\begin{aligned}
& {[\mu x, \mu y]=i \varepsilon\left(1-\mu^{\prime} z\right),} \\
& {\left[\mu x, \mu^{\prime} z\right]=i \varepsilon\left(\mu y \mu^{\prime} z+\mu^{\prime} z \mu y\right),} \\
& {\left[\mu y, \mu^{\prime} z\right]=-i \varepsilon\left(\mu x \mu^{\prime} z+\mu^{\prime} z \mu x\right) .}
\end{aligned}
$$

Constant $\varepsilon$ in (2.1) is a dimensionless parameter while $\mu$ and $\mu^{\prime}$ have dimension of mass or of inverse length and they describe 'magnitudes' of the $x-y$ and $z$ directions respectively. Clearly, $\varepsilon=0$ describes the 'commutative limit' of (2.1). Contraction $\mu^{\prime} \rightarrow 0$ on the other hand gives the Heisenberg algebra; in a way, $\mu^{\prime} \rightarrow 0$ is a subspace $z=0$ of truncated Heisenberg space (2.1). For $\varepsilon=1$ and $\mu^{\prime}=\mu$ the algebra has finite-dimensional representations by $n \times n$ matrices for any $n$ :

$$
\begin{aligned}
& x=\frac{1}{\mu \sqrt{2}}\left(\begin{array}{cccccc}
0 & 1 & 0 & . & . & . \\
1 & 0 & \sqrt{2} & . & . & . \\
0 & \sqrt{2} & 0 & . & . & . \\
. & . & . & . & . & . \\
. & . & . & . & 0 & \sqrt{n-1} \\
. & . & . & . & \sqrt{n-1} & 0
\end{array}\right), \\
& y=\frac{i}{\mu \sqrt{2}}\left(\begin{array}{cccccc}
0 & -1 & 0 & . & . & . \\
1 & 0 & -\sqrt{2} & . & . & . \\
0 & \sqrt{2} & 0 & . & . & . \\
. & . & . & . & . & . \\
. & . & . & . & 0 & -\sqrt{n-1} \\
. & . & . & . & \sqrt{n-1} & 0
\end{array}\right) \text {, }
\end{aligned}
$$




$$
z=\frac{n}{\mu}\left(\begin{array}{lllll}
0 & 0 & 0 & . & . \\
0 & 0 & 0 & . & . \\
0 & 0 & 0 & . & . \\
. & . & . & . & . \\
. & . & . & 0 & 0 \\
. & . & . & 0 & 1
\end{array}\right) .
$$

The limit $z \rightarrow 0$ is a weak limit from finite matrices to the infinite-matrix representation of the Heisenberg algebra; as we shall shortly show, $z=0$ can also be treated as a subspace in a consistent geometric way.

\section{Differential geometry, forms}

Differential geometry which corresponds to a given noncommutative algebra can be defined in various ways. We choose to work in the noncommutative frame formalism, [6], in particular because it is well adjusted to matrix geometries. Its basic ingredients are the following:

- Cotangent space which has the basis of 1 -forms $\theta^{\alpha}$, the frame.

- Locally flat (inverse) metric: $g^{\alpha \beta}=g\left(\theta^{\alpha} \otimes \theta^{\beta}\right)=\eta^{\alpha \beta}$;

condition $\left[f, \theta^{\alpha}\right]=0$ is imposed to insure local flatness.

- Derivations $e_{\alpha}$ dual to $\theta^{\alpha}$ which satisfy the Leibniz rule;

all $e_{\alpha}$ are inner, defined by momenta $p_{\alpha}$ which belong to the algebra: $e_{\alpha} f=\left[p_{\alpha}, f\right]$.

- Differential $d$ defined in terms of the frame, $d f=\left(e_{\alpha} f\right) \theta^{\alpha}$.

- All mappings are (bi)linear.

Clearly in this setting differential is defined by momenta and thus by choosing different sets of momenta $p_{\alpha}$ we obtain different differentials. However the choice is not completely arbitrary. Imposing the Leibniz rule and condition $d^{2}=0$ we find that the momenta satisfy quadratic algebra

$$
\left[p_{\alpha}, p_{\beta}\right]=\frac{1}{i \varepsilon} K_{\alpha \beta}+F_{\alpha \beta}^{\gamma} p_{\gamma}-2 i \varepsilon Q_{\alpha \beta}^{\gamma \delta} p_{\gamma} p_{\delta}
$$

which perhaps is the most restictive constraint in the frame formalism. It allows on the other hand to develop the formalism in many details. Constants $K_{\alpha \beta}, F^{\gamma}{ }_{\alpha \beta}$ and $Q^{\gamma \delta}{ }_{\alpha \beta}$ are called the structure coefficients.

Exterior algebra has to be consistent with the differential. If we define the exterior multiplication of two frame 1 -forms by

$$
\theta^{\gamma} \theta^{\delta}=P_{\alpha \beta}^{\gamma \delta} \theta^{\alpha} \otimes \theta^{\beta}
$$

this consistency implies that

$$
P_{\alpha \beta}^{\gamma \delta}=\frac{1}{2}\left(\delta_{\alpha}^{\gamma} \delta_{\beta}^{\delta}-\delta_{\beta}^{\gamma} \delta_{\alpha}^{\delta}\right)+i \varepsilon Q_{\alpha \beta}^{\gamma \delta}
$$


Truncated Heisenberg algebra (2.1) is already quadratic in its generators, so the momenta can be introduced as

$$
\varepsilon p_{1}=i \mu^{2} y, \quad \varepsilon p_{2}=-i \mu^{2} x, \quad \varepsilon p_{3}=i \mu\left(\mu z-\frac{1}{2}\right) .
$$

The $p_{\alpha}$ are chosen in such way that for $z=0, p_{1}$ and $p_{2}$ reduce to the momenta usually defined in the two-dimensional flat space. The momentum algebra is

$$
\begin{aligned}
& {\left[p_{1}, p_{2}\right]=\frac{\mu^{2}}{2 i \varepsilon}+\mu p_{3},} \\
& {\left[p_{2}, p_{3}\right]=\mu p_{1}-i \varepsilon\left(p_{1} p_{3}+p_{3} p_{1}\right),} \\
& {\left[p_{3}, p_{1}\right]=\mu p_{2}-i \varepsilon\left(p_{2} p_{3}+p_{3} p_{2}\right),}
\end{aligned}
$$

and we can identify the structure coefficients:

$$
K_{12}=\frac{\mu^{2}}{2}, \quad F_{23}^{1}=\mu, \quad Q^{13}{ }_{23}=\frac{1}{2}, \quad Q^{23}{ }_{31}=\frac{1}{2} .
$$

As we have seen, the exterior algebra is completely defined by (3.5). As a basis of 2-forms we can take for example the anticommutators if 1-forms. The basic relations in the exterior algebra are:

$$
\begin{aligned}
& \left(\theta^{1}\right)^{2}=0, \quad\left(\theta^{2}\right)^{2}=0, \quad\left(\theta^{3}\right)^{2}=0, \\
& \left\{\theta^{1}, \theta^{2}\right\}=0, \\
& \left\{\theta^{1}, \theta^{3}\right\}=i \varepsilon\left(\theta^{2} \theta^{3}-\theta^{3} \theta^{2}\right), \\
& \left\{\theta^{2}, \theta^{3}\right\}=i \varepsilon\left(\theta^{3} \theta^{1}-\theta^{1} \theta^{3}\right) .
\end{aligned}
$$

The space of 2-forms is three-dimensional; as we can see, due to quadratic terms in (3.1), 1-forms do not anticommute.

The algebra of 3-forms is obtained from the algebra of 2-forms and associativity:

$$
\begin{aligned}
& \theta^{1} \theta^{3} \theta^{1}=\theta^{2} \theta^{3} \theta^{2} \\
& \theta^{1} \theta^{2} \theta^{3}=-\theta^{2} \theta^{1} \theta^{3}=\theta^{3} \theta^{1} \theta^{2}=-\theta^{3} \theta^{2} \theta^{1}=i \frac{\varepsilon^{2}-1}{2 \varepsilon} \theta^{2} \theta^{3} \theta^{2} \\
& \theta^{1} \theta^{3} \theta^{2}=-\theta^{2} \theta^{3} \theta^{1}=i \frac{\varepsilon^{2}+1}{2 \varepsilon} \theta^{2} \theta^{3} \theta^{2} . \\
& \theta^{3} \theta^{1} \theta^{3}=0, \quad \theta^{3} \theta^{2} \theta^{3}=0 .
\end{aligned}
$$

These relations mean that there is only one linearly independent 3-form, which implies that the volume form is well defined. We choose it as $\Theta=-\frac{i}{2 \varepsilon} \theta^{2} \theta^{3} \theta^{2}$. 


\section{Differential geometry, curvature}

Let us denote by $C_{\beta \gamma}^{\alpha}$ the Ricci rotation coefficients:

$$
d \theta^{\alpha}=-\frac{1}{2} C_{\beta \gamma}^{\alpha} \theta^{\beta} \theta^{\gamma}
$$

From the general formalism, [6], it follows that the Ricci rotation coefficients are linear in the momenta. In terms of the structure coefficients they are given by

$$
C_{\alpha \beta}^{\gamma}=F_{\alpha \beta}^{\gamma}-4 i \varepsilon Q^{\gamma \delta}{ }_{\alpha \beta} p_{\delta}
$$

Main quantities which describe geometry of noncommutative spaces can be defined in complete analogy with commutative geometry. This generalization in the noncommutative frame formalism includes the requirement of linearity, a notion natural but in effect quite restrictive. We recall the main results.

The inverse metric $g$ in the frame basis has constant components

$$
g^{\alpha \beta}=g\left(\theta^{\alpha} \otimes \theta^{\beta}\right)=\text { const. }
$$

Usually we take it to be $g^{\alpha \beta}=\eta^{\alpha \beta}$ or $g^{\alpha \beta}=\delta^{\alpha \beta}$. The metric is a mapping bilinear in its arguments; from bilinearity one can calculate the coordinate components. Denoting

$$
e_{\alpha}^{\mu}(x)=\left[p_{\alpha}, x^{\mu}\right]
$$

which is a natural notation as we have already defined

$$
d x^{\mu}=\left[p_{\alpha}, x^{\mu}\right] \theta^{\alpha}=e_{\alpha}^{\mu} \theta^{\alpha},
$$

we obtain

$$
g^{\mu v}=g\left(d x^{\mu} \otimes d x^{v}\right)=e_{\alpha}^{\mu} g\left(\theta^{\alpha} \otimes \theta^{\beta}\right) e_{\beta}^{v}=e_{\alpha}^{\mu} e_{\beta}^{v} \eta_{\alpha \beta},
$$

because we have assumed that the frame commutes with all functions so $e_{\beta}^{v} \theta^{\beta}=\theta^{\beta} e_{\beta}^{v}$. Of course, we obtain that $g^{\mu v}=g^{\mu v}(x)$ and $f d x^{\mu} \neq d x^{\mu} f$ in general.

The connection $\omega^{\alpha}{ }_{\beta}=\omega_{\gamma \beta}^{\alpha} \theta^{\gamma}$ is a 1-form which defines the covariant derivative; the torsion $\Theta^{\alpha}=\Theta^{\alpha}{ }_{\gamma \beta} \theta^{\gamma} \theta^{\beta}$ is a 2-form and they are related by the structure equation $\Theta^{\alpha}=d \theta^{\alpha}+\omega^{\alpha}{ }_{\beta} \theta^{\beta}$. Conditions of the metric compatibility of connection and of vanishing of the torsion have been discussed in [6] and solved in important cases. When noncommutativity parameter $\varepsilon$ is small, a metric compatible connection can be defined by the usual relation

$$
\omega_{\alpha \beta \gamma}=\frac{1}{2}\left(C_{\alpha \beta \gamma}-C_{\beta \gamma \alpha}+C_{\gamma \alpha \beta}\right) .
$$

For the truncated Heisenberg space we find

$$
\begin{aligned}
& C^{1}{ }_{23}=-C_{32}^{1}=2 \mu^{2} z, \quad C^{2}{ }_{31}=-C_{31}^{2}=2 \mu^{2} z, \quad C^{3}{ }_{12}=-C^{3}{ }_{21}=\mu, \\
& C^{3}{ }_{13}=-C_{31}^{3}=2 \mu^{2} x, \quad C^{3}{ }_{23}=-C^{3}{ }_{32}=2 \mu^{2} y,
\end{aligned}
$$


so we define the connection as

$$
\begin{aligned}
& \omega_{12}=-\omega_{21}=\left(-\frac{\mu}{2}+2 i \varepsilon p_{3}\right) \theta^{3}=\mu\left(\frac{1}{2}-2 \mu z\right) \theta^{3}, \\
& \omega_{13}=-\omega_{31}=\frac{\mu}{2} \theta^{2}+2 i \varepsilon p_{2} \theta^{3}=\frac{\mu}{2} \theta^{2}+2 \mu^{2} x \theta^{3}, \\
& \omega_{23}=-\omega_{32}=-\frac{\mu}{2} \theta^{1}-2 i \varepsilon p_{1} \theta^{3}=-\frac{\mu}{2} \theta^{1}+2 \mu^{2} y \theta^{3} .
\end{aligned}
$$

The connection defines the Riemann curvature tensor $\Omega^{\alpha}{ }_{\beta}$

$$
\Omega_{\beta}^{\alpha}=d \omega_{\beta}^{\alpha}+\omega_{\gamma}^{\alpha} \omega_{\beta}^{\gamma}=R_{\beta \rho \sigma}^{\alpha} \theta^{\rho} \theta^{\sigma} .
$$

Obviously, given the connection and the Ricci coefficients we can calculate the curvature. Using (4.7) we obtain for $\Omega^{\alpha}{ }_{\beta}$ an expression quadratic in the momenta, [7],

$$
\begin{aligned}
& R_{\beta \rho \sigma}^{\alpha} \theta^{\rho} \theta^{\sigma}=2\left(T_{\sigma \beta}^{\alpha \gamma} K_{\rho \gamma}-\frac{1}{4} F_{\gamma \beta}^{\alpha} F_{\rho \sigma}^{\gamma}+\frac{1}{4} F_{\rho \gamma}^{\alpha}{ }_{\sigma \beta}^{\gamma}\right. \\
& \quad+i \varepsilon p_{\zeta}\left(F_{\rho \gamma}^{\zeta} T_{\sigma \beta}^{\alpha \gamma}+F_{\gamma \beta}^{\alpha} Q_{\rho \sigma}^{\gamma \zeta}-\frac{1}{2} F_{\rho \sigma}^{\gamma} T_{\gamma \beta}^{\alpha \zeta}+\frac{1}{2} F_{\rho \gamma}^{\alpha} T_{\sigma \beta}^{\gamma \zeta}+\frac{1}{2} F_{\sigma \beta}^{\gamma} T_{\rho \gamma}^{\alpha \zeta}\right) \\
& \left.\quad+(i \varepsilon)^{2} p_{\zeta} p_{\eta}\left(-2 T_{\sigma \beta}^{\alpha \gamma} Q_{\rho \gamma}^{\zeta \eta}+2 T_{\gamma \beta}^{\alpha \zeta} Q_{\rho \sigma}^{\gamma \eta}+T_{\rho \gamma}^{\alpha \zeta} T_{\sigma \beta}^{\gamma \eta}\right)\right) \theta^{\rho} \theta^{\sigma},
\end{aligned}
$$

where coefficients $T_{\alpha \beta \gamma \delta}$ are defined by

$$
T_{\alpha \beta \gamma \delta}=2\left(-Q_{\alpha \beta \gamma \delta}+Q_{\beta \gamma \delta \alpha}+Q_{\beta \delta \gamma \alpha}\right) .
$$

From the Riemann curvature we can define the Ricci curvature, $R_{\alpha \beta}=R_{\alpha \gamma \beta}^{\gamma}$, and the curvature scalar, $R=\eta^{\alpha \beta} R_{\alpha \beta}$. For the truncated Heisenberg space we obtain for $R$ a simple expression

$$
R=\frac{11}{4} \mu^{2}-2 \mu^{2}\left(\mu z-\frac{1}{2}\right)-4 \mu^{4}\left(x^{2}+y^{2}\right) .
$$

It is perhaps also interesting to write explicitly the Ricci tensor reduced to subspace $z=0$ :

$$
R_{\alpha \beta}=\left(\begin{array}{ccc}
\frac{3 \mu^{2}}{2}-4 \mu^{4} x^{2} & -2 \mu^{4}(x y+y x)+i \frac{\varepsilon \mu^{2}}{4} & 2 \mu^{3} y+2 i \varepsilon \mu^{3} x \\
-2 \mu^{4}(x y+y x)-i \frac{\varepsilon \mu^{2}}{4} & \frac{3 \mu^{2}}{2}-4 \mu^{4} y^{2} & -2 \mu^{3} x+2 i \varepsilon \mu^{3} y \\
2 \mu^{3} y-2 i \varepsilon \mu^{3} x & -2 \mu^{3} x-2 i \varepsilon \mu^{3} y & \frac{9 \mu^{2}}{2}-4 \mu^{4}\left(x^{2}+y^{2}\right)
\end{array}\right) .
$$

The range of the frame indices, $\alpha=1,2,3$ indicates the fact that, although the truncated Heisenberg space reduced to $z=0$ is two-dimensional, the cotangent space it remains to be three-dimensional. 


\section{Scalar field}

Having the value of the scalar curvature (4.13) it is not difficult to recognize the relation between the Grosse-Wulkenhaar action (1.1) and the action for the scalar field on a curved space. In the notation of [3], $\mathrm{x}_{\mu}=i p_{\mu}$ and we have $\mathrm{x}^{\mu} \mathrm{x}_{\mu}=-\frac{\mu^{4}}{\varepsilon^{2}} x^{\mu} x_{\mu}$. The Grosse-Wulkenhaar action can be rewritten as

$$
S=\int \frac{1}{2}\left(1-\frac{\Omega^{2}}{2}\right) \partial_{\mu} \varphi \partial^{\mu} \varphi+\frac{m^{2}}{2} \varphi^{2}+\frac{\Omega^{2}}{2} x^{\mu} x_{\mu} \varphi \varphi+\frac{\lambda}{4 !} \varphi^{4} .
$$

The action for the scalar field coupled to the curvature of the background space on the other hand is given by

$$
S^{\prime}=\int \frac{1}{2} e_{\alpha} \varphi e^{\alpha} \varphi+\frac{M^{2}}{2} \varphi^{2}-\frac{\xi}{2} R \varphi^{2}+\frac{\Lambda}{4 !} \varphi^{4} .
$$

As we have seen already, for $z=0, e_{\alpha}=\delta_{\alpha}^{\mu} \partial_{\mu}(\mu=1,2)$ and $e_{3}=0$. Therefore we find that (5.1) and (5.2) are the same up to an overall rescaling

$$
S=\kappa S^{\prime}
$$

if we identify

$$
1-\frac{\Omega^{2}}{2}=\kappa, \quad m^{2}=\kappa\left(M^{2}-\xi a\right), \quad \frac{\Omega^{2} \mu^{4}}{\varepsilon^{2}}=\kappa \xi b, \quad \lambda=\kappa \Lambda,
$$

where $a=15 \mu^{2} / 2, b=8 \mu^{4}$.

Remarkably, the Grosse-Wulkenhaar action has a simple geometric interpretation: it describes a scalar field moving on a curved background. The constant part of the curvature as always renormalizes the mass, while the space-dependent part gives exactly the harmonic oscillator potential. The coupling constant $\xi$ is not a priori fixed but it can be related to $\Omega$. If we identify the two actions at the self-duality point $\Omega=1$ we obtain

$$
\xi=\frac{\Omega^{2} \mu^{4}}{\varepsilon^{2} \kappa b}=\frac{1}{4 \varepsilon^{2}} .
$$

\section{Gauge fields}

Since the formalism is defined geometrically it is well adapted to describe gauge symmetries. We shall discuss noncommutative $U_{1}$ symmetry. The corresponding gauge fields are defined as usual: the vector potential $A$ is a 1-form, the field strength $F$ is a 2-form:

$$
A=A_{\alpha} \theta^{\alpha}, \quad F=d A+A^{2}=\frac{1}{2} F_{\alpha \beta} \theta^{\alpha} \theta^{\beta} .
$$

Both are taken to be antihermitian. Components of the field strength satisfy $F_{\zeta \eta}=F_{\alpha \beta} P^{\alpha \beta}{ }_{\zeta \eta}$, which in our case means that they are antisymmetric in indices $\zeta$ and $\eta$. We find

$$
F_{\zeta \eta}=e_{[\zeta} A_{\eta]}-A_{\alpha} C_{\zeta \eta}^{\alpha}+\left[A_{\zeta}, A_{\eta}\right]+2 i \varepsilon\left(e_{\beta} A_{\gamma}\right) Q^{\beta \gamma}{ }_{\zeta \eta}+2 i \varepsilon A_{\beta} A_{\gamma} Q^{\beta \gamma}{ }_{\zeta \eta}
$$

When the calculus is based on inner derivations there is a special connection 1-form $\theta$,

$$
\theta=-p_{\alpha} \theta^{\alpha}
$$


Differential can be expressed as $d f=-[\theta, f]$ and, as one can show easily,

$$
d \theta+\theta^{2}=\frac{1}{2 i \varepsilon} K_{\alpha \beta} \theta^{\alpha} \theta^{\beta} .
$$

$\theta$ is invariant under the action of the gauge group: one can see it from

$$
\theta^{\prime}=g^{-1} \theta g+g^{-1} d g
$$

and

$$
g^{-1} \theta g=g^{-1}[\theta, g]+g^{-1} g \theta=-g^{-1} d g+\theta .
$$

Difference between connections $A$ and $\theta, \mathrm{X}=A-\theta=\mathrm{X}_{\alpha} \theta^{\alpha}, \mathrm{X}_{\alpha}=p_{\alpha}+A_{\alpha}$, is called the covariant coordinate. As a difference of two connections it transforms covariantly, in the adjoint representation of the gauge group. Expressing the field strength in $\mathrm{X}_{\alpha}$ we obtain

$$
F_{\alpha \beta}=2 P_{\alpha \beta}^{\gamma \delta} \mathrm{X}_{\gamma} \mathrm{X}_{\delta}-F_{\alpha \beta}^{\gamma} \mathrm{X}_{\gamma}-\frac{1}{i \varepsilon} K_{\alpha \beta} .
$$

Taking into account the value of the Ricci rotation coefficients, for the components of the field strength on the truncated Heisenberg space we have

$$
\begin{aligned}
& \mathrm{F}_{12}=e_{1} \mathrm{~A}_{2}-e_{2} \mathrm{~A}_{1}+\left[\mathrm{A}_{1}, \mathrm{~A}_{2}\right]-\mu \mathrm{A}_{3}, \\
& \mathrm{~F}_{13}=\left[p_{1}+\mathrm{A}_{1}, \mathrm{~A}_{3}\right]-i \varepsilon\left\{p_{2}+\mathrm{A}_{2}, \mathrm{~A}_{3}\right\}+2 \mu^{2} \mathrm{~A}_{2} z, \\
& \mathrm{~F}_{23}=\left[p_{2}+\mathrm{A}_{2}, \mathrm{~A}_{3}\right]+i \varepsilon\left\{p_{1}+\mathrm{A}_{1}, \mathrm{~A}_{3}\right\}-2 \mu^{2} \mathrm{~A}_{1} z .
\end{aligned}
$$

We wish to reduce the gauge field from three-dimensional space to a two-dimensional subspace. For $z=0, p_{3}=-\frac{i \mu}{2 \varepsilon}, e_{3}=0$ and the component $A_{3}$ of the potential transforms as a scalar field in the adjoint representation. We denote

$$
\mathrm{A}_{3}=\phi, \quad \mathrm{A}_{1}=A_{1}, \quad \mathrm{~A}_{2}=A_{2}
$$

that is,

$$
\mathrm{X}_{1}=p_{1}+A_{1}, \quad \mathrm{X}_{2}=p_{2}+A_{2}, \quad \mathrm{X}_{3}=-\frac{i \mu}{2 \varepsilon}+\phi .
$$

Were the gauge fields defined intrinsically on the two-dimensional plane we would have

$$
\begin{aligned}
& D_{\alpha} \phi=\left[p_{\alpha}+A_{\alpha}, \phi\right]=e_{\alpha} \phi+\left[A_{\alpha}, \phi\right], \\
& F_{12}=e_{1} A_{2}-e_{2} A_{1}+\left[A_{1}, A_{2}\right],
\end{aligned}
$$

but on the dimensionally reduced space we obtain

$$
\begin{aligned}
& \mathrm{F}_{12}=F_{12}-\mu \phi=\left[\mathrm{X}_{1}, \mathrm{X}_{2}\right]+\frac{i \mu^{2}}{\varepsilon}-\mu \phi \\
& \mathrm{F}_{13}=D_{1} \phi-i \varepsilon\left\{p_{2}+A_{2}, \phi\right\}=\left[\mathrm{X}_{1}, \phi\right]-i \varepsilon\left\{\mathrm{X}_{2}, \phi\right\} \\
& \mathrm{F}_{23}=D_{2} \phi+i \varepsilon\left\{p_{1}+A_{1}, \phi\right\}=\left[\mathrm{X}_{2}, \phi\right]+i \varepsilon\left\{\mathrm{X}_{1}, \phi\right\}
\end{aligned}
$$




\section{Action}

To write the Yang-Mills action we need the symmetrized product of forms,

$$
\mathscr{S}_{Y M}=\frac{1}{16} \int\left(\mathrm{F}^{*} \mathrm{~F}+{ }^{*} \mathrm{FF}\right)
$$

where the Hodge-dual is defined as

$$
*\left(\frac{1}{2}\left[\theta^{1}, \theta^{2}\right]\right)=\theta^{3}, \quad *\left(\frac{1}{2}\left[\theta^{2}, \theta^{3}\right]\right)=\theta^{1}, \quad *\left(\frac{1}{2}\left[\theta^{3}, \theta^{1}\right]\right)=\theta^{2} .
$$

This definition modifies the usual normalization and gives for example

$$
*\left(\frac{1}{2}\left[\theta^{1}, \theta^{2}\right]\right) \frac{1}{2}\left[\theta^{1}, \theta^{2}\right]=\left(1-\varepsilon^{2}\right) \Theta .
$$

For $\varepsilon=0$ action (7.1) reduces to the commutative expression, while in our case

$$
\mathscr{S}_{Y M}=\frac{1}{2} \operatorname{Tr}\left(\left(1-\varepsilon^{2}\right) \mathrm{F}_{12} \mathrm{~F}^{12}+\mathrm{F}_{13} \mathrm{~F}^{13}+\mathrm{F}_{23} \mathrm{~F}^{23}\right) .
$$

Writing the fields in components we obtain

$$
\begin{aligned}
\mathscr{S}_{Y M}= & \frac{1}{2} \operatorname{Tr}\left(\left(1-\varepsilon^{2}\right)\left(F_{12}\right)^{2}-2\left(1-\varepsilon^{2}\right) \mu F_{12} \phi+\left(5-\varepsilon^{2}\right) \mu^{2} \phi^{2}+4 i \varepsilon F_{12} \phi^{2}\right. \\
& \left.+\left(D_{1} \phi\right)^{2}+\left(D_{2} \phi\right)^{2}-\varepsilon^{2}\left\{p_{1}+A_{1}, \phi\right\}^{2}-\varepsilon^{2}\left\{p_{2}+A_{2}, \phi\right\}^{2}\right),
\end{aligned}
$$

or in covariant coordinates

$$
\begin{aligned}
\mathscr{S}_{Y M}= & \frac{1}{2} \operatorname{Tr}\left(\left(1-\varepsilon^{2}\right)\left(\left[\mathrm{X}_{1}, \mathrm{X}_{2}\right]^{2}+\mu^{2} \phi^{2}-\frac{2 i \mu^{3}}{\varepsilon} \phi-2 \mu\left[\mathrm{X}_{1}, \mathrm{X}_{2}\right] \phi\right)\right. \\
& \left.+4 i \varepsilon\left[\mathrm{X}_{1}, \mathrm{X}_{2}\right] \phi^{2}+\left[\mathrm{X}_{1}, \phi\right]^{2}+\left[\mathrm{X}_{2}, \phi\right]^{2}-\varepsilon^{2}\left\{\mathrm{X}_{1}, \phi\right\}^{2}-\varepsilon^{2}\left\{\mathrm{X}_{2}, \phi\right\}^{2}\right) .
\end{aligned}
$$

Equations of motion corresponding to this action are given by

$$
\begin{aligned}
& D^{\alpha} D_{\alpha} \phi+\varepsilon^{2}\left\{p^{\alpha}-A^{\alpha},\left\{p_{\alpha}+A_{\alpha}, \phi\right\}\right\}+\left(1-\varepsilon^{2}\right) \mu F_{12}-\left(5-\varepsilon^{2}\right) \mu^{2} \phi-2 i \varepsilon\left\{F_{12}, \phi\right\}=0, \\
& \left(1-\varepsilon^{2}\right) \varepsilon^{\alpha \beta} D_{\beta}\left(F_{12}-\mu \phi\right)+2 i \varepsilon \varepsilon^{\alpha \beta}\left\{D_{\beta} \phi, \phi\right\}-\left[D_{\alpha} \phi, \phi\right]-\varepsilon^{2}\left\{\left\{p^{\alpha}+A^{\alpha}, \phi\right\}, \phi\right\}=0 .
\end{aligned}
$$

It is difficult to find their general solution, but when we confine to constant solutions we obtain the following two:

$$
\begin{array}{ll}
A_{1}=0, & A_{2}=0, \quad \phi=0, \\
\mathrm{X}_{1}=0, & \mathrm{X}_{2}=0,
\end{array}
$$

The first solution is the usual vacuum.

Due to the properties of exterior multiplication, the only 'viable' Chern-Simons action is

$$
\mathscr{S}_{C S}=\alpha \int \mathrm{X}^{3}=\alpha \int \mathrm{X}_{\alpha} \mathrm{X}_{\beta} \mathrm{X}_{\gamma} \Delta_{\zeta \eta \xi}^{\alpha \beta \gamma} \theta^{\zeta} \theta^{\eta} \theta^{\xi}
$$

where noncommutative $\Delta_{\zeta \eta \xi}^{\alpha \beta \gamma}$ is a generalization of the commutative fully antisymmetric tensor $\delta_{\zeta \eta \xi}^{\alpha \beta \gamma},[5]$, or in components

$$
\mathscr{S}_{C S}=\frac{\alpha \mu}{3} \operatorname{Tr}\left(\left(3-\varepsilon^{2}\right)\left[\mathrm{X}_{1}, \mathrm{X}_{2}\right] \mathrm{X}_{3}+2 i \varepsilon\left(\mathrm{X}_{1}^{2}+\mathrm{X}_{2}^{2}\right) \mathrm{X}_{3}\right) \text {. }
$$

The Chern-Simons action can be included in the classical and in the quantum action, but as it does not simplify the equations of motion or the quantization significantly, we will omit it. 


\section{BRST invariance}

According to the usual procedure, the quantum action is

$$
\mathscr{S}=\mathscr{S}_{Y M}+\mathscr{S}_{g f}
$$

The simplest gauge-fixing term is given by

$$
\mathscr{G}=e_{\alpha} A^{\alpha}=\partial_{1} A^{1}+\partial_{2} A^{2}
$$

and it can be written in terms of the ghost $c$, the antighost $\bar{c}$ and the auxilliary field $B$ as

$$
\mathscr{S}_{g f}=\operatorname{Tr}\left(B \mathscr{G}+\frac{\alpha}{2} B B-\bar{c} e_{\alpha} D^{\alpha} c\right) .
$$

A nilpotent BRST transformation $s$ can be introduced in a familiar way

$$
\begin{aligned}
& s A_{\alpha}=D_{\alpha} c=e_{\alpha} c+i\left[A_{\alpha}, c\right], \\
& s F_{\alpha \beta}=\left[F_{\alpha \beta}, c\right], \\
& s \phi=[\phi, c], \\
& s\left(\mathrm{X}_{\alpha}\right)=\left[\mathrm{X}_{\alpha}, c\right]=e_{\alpha} c+i\left[A_{\alpha}, c\right]=s A_{\alpha}, \\
& s c=-c^{2}, \\
& s \bar{c}=B, \\
& s B=0 .
\end{aligned}
$$

As $\mathscr{S}_{g f}=\operatorname{Tr} s\left(\bar{c} \mathscr{G}+\frac{\alpha}{2} \bar{c} B\right)$, the BRST transformation leaves the quantum action (8.1) invariant.

\section{Summary}

Our aim in this talk was to describe and analyze possible relations between renormalizability, noncommutative gravity and matrix models. The initial idea was to explain the fact that the renormalizable Grosse-Wulkenhaar model is a model of scalar field on a curved noncommutative space. The space itself is closely related to the oscillator representation of coordinate and momentum in quantum mechanics, or more precisely, to its finite matrix truncations.

Truncated Heisenberg space has highly nontrivial geometry. It is three-dimensional and axially symetric (around the $z$-axis; the generator of rotations is $M=\frac{1}{2}\left((\mu x)^{2}+(\mu y)^{2}+\mu^{\prime} z\right)$ ). The space is curved and components of the curvature tensors can be calculated. The exterior algebra has interesting properties: it is well defined but quite different from the corresponding commutative algebra: For example, for value of noncommutativity parameter $\varepsilon=1$, the rules of multiplication imply that $\theta^{1} \theta^{2} \theta^{3}=0$ while at the same time $\theta^{2} \theta^{3} \theta^{2} \neq 0$ which is the reason why we choose the latter to define the volume form.

For the same value $\varepsilon=1$ the truncated Heisenberg algebra has finite representations, while the contraction $\mu^{\prime} \rightarrow 0$ gives the Heisenberg algebra with its infinite-dimensional representation. We use this contraction, or dimensional reduction, to define models of scalar and gauge fields. 
We discuss in some detail how to construct classical $U_{1}$ gauge theory following the same noncommutative-geometric logic as for the scalar field, in order to obtain a candidate for renormalizable gauge theory. As a result we obtain the action

$$
\begin{aligned}
\mathscr{S}_{Y M}= & \frac{1}{2} \int\left(\left(1-\varepsilon^{2}\right)\left(F_{12}\right)^{2}-2\left(1-\varepsilon^{2}\right) \mu F_{12} \phi+\left(5-\varepsilon^{2}\right) \mu^{2} \phi^{2}+4 i \varepsilon F_{12} \phi^{2}\right. \\
& \left.+\left(D_{1} \phi\right)^{2}+\left(D_{2} \phi\right)^{2}-\varepsilon^{2}\left\{p_{1}+A_{1}, \phi\right\}^{2}-\varepsilon^{2}\left\{p_{2}+A_{2}, \phi\right\}^{2}\right)
\end{aligned}
$$

which consists of a gauge field and a scalar field coupled in a particular way. Both fields propagate except in the case $\varepsilon=1$, in which the action reduces to

$$
\left.\mathscr{S}_{Y M}\right|_{\mathcal{E}=1}=\frac{1}{2} \int\left(D_{1} \phi\right)^{2}+\left(D_{2} \phi\right)^{2}+4 \mu^{2} \phi^{2}+4 i F_{12} \phi^{2}-\left\{p_{1}+A_{1}, \phi\right\}^{2}-\left\{p_{2}+A_{2}, \phi\right\}^{2} .
$$

In general, this action implies mixed gauge-scalar propagators but all of them are related to or expressible in terms of the Mehler kernel, [8].

The next step would be to quantize the presented model. Preliminary results in this direction are encouraging: First, the model has as a solution to the equations of motion the usual vacuum $A_{1}=0, A_{2}=0, \phi=0$. Further, a nilpotent BRST transformation $s$ can be defined and it leaves the quantum action invariant. The 'only' remaining thing to perform is the explicit quantization, and this we will try to do in our future work.

\section{References}

[1] H. S. Snyder, Quantized spacetime, Phys.Rev. 71, 38 (1947).

[2] H. Grosse and R. Wulkenhaar, JHEP 0312, 019 (2003) [arXiv:hep-th/0307017].

[3] H. Grosse and R. Wulkenhaar, Commun. Math. Phys. 256, 305 (2005) [arXiv:hep-th/0401128],

H. Grosse and R. Wulkenhaar, Lett. Math. Phys. 71 (2005) 13.

[4] E. Langmann and R. J. Szabo, Phys. Lett. B 533 (2002) 168 [arXiv:hep-th/0202039].

[5] M. Buric, H. Grosse and J. Madore, JHEP 1007 (2010) 010 [arXiv:1003.2284 [hep-th]].

[6] J. Madore, "An Introduction To Noncommutative Differential Geometry And Its Physical Applications," Lond. Math. Soc. Lect. Note Ser. 257 (2000) 1.

[7] M. Buric and M. Wohlgenannt, JHEP 1003 (2010) 053 [arXiv:0902.3408 [hep-th]].

[8] H. Grosse and M. Wohlgenannt, Eur. Phys. J. C 52, 435 (2007) [arXiv:hep-th/0703169]. 